\title{
ON THE CAUCHY PROBLEM FOR THE NONLOCAL DERIVATIVE NONLINEAR SCHRÖDINGER EQUATION *
}

\author{
ROGER PERES DE MOURA ${ }^{\dagger}$ AND ADEMIR PASTOR ${ }^{\ddagger}$
}

\begin{abstract}
We consider the Cauchy problem associated with the one-dimensional nonlocal derivative nonlinear Schrödinger equation, and establish local well-posedness for "small" initial data in the usual $L^{2}$-based Sobolev spaces $H^{s}(\mathbb{R}), s>1 / 2$. We also prove that our result is "almost sharp" in the sense that the flow-map data-solution fails to be $C^{3}$ at the origin from $H^{s}(\mathbb{R})$ to $H^{s}(\mathbb{R})$ for any $s<1 / 2$. Finally, thanks to the lack of energy conservation, we prove the nonexistence of solitary-wave solutions.
\end{abstract}

Key words. Nonlocal derivative nonlinear Schrödinger equation, Cauchy problem, wellposedness, solitary waves.

AMS subject classifications. 35A01, 35C07, 35G25, 35Q55.

\section{Introduction}

The derivative nonlinear Schrödinger (DNLS) equation

$$
\partial_{t} u-i \partial_{x}^{2} u=\partial_{x}\left(|u|^{2} u\right), \quad x, t \in \mathbb{R},
$$

has been derived for several physical models in propagation of nonlinear waves; for example, in the study of (i) drifting filamentations formed in nonlinear waves in magnetized plasmas, (ii) the evolution of light pulses in optical fibers, and (iii) weakly nonlinear and dispersive Alfvén waves in a plasma (see e.g. [19, 20]). However, in the last example, if effects of resonant particles on the Alfvén waves modulations are included, one obtains the nonlocal derivative nonlinear Schrödinger (nDNLS) equation, which can be written, in dimensionless variables, as (see e.g. $[8,20]$ )

$$
\partial_{t} u-i \partial_{x}^{2} u=\partial_{x}\left(|u|^{2} u\right)+\lambda \partial_{x}\left(\mathcal{H}\left(|u|^{2}\right) u\right), \quad x, t \in \mathbb{R} .
$$

Here $u=u(x, t)$ is a complex-valued function, $\lambda$ is a real parameter depending on the velocity distributions of the particle species, and $\mathcal{H}$ is the Hilbert transform defined by

$$
(\mathcal{H} f)(x)=\text { p.v. } \frac{1}{\pi} \int_{-\infty}^{+\infty} \frac{f(y)}{x-y} d y,
$$

where p.v. denotes the Cauchy principal value.

The nonlocal term $\lambda \partial_{x}\left(\mathcal{H}\left(|u|^{2}\right) u\right)$ in (1.2) represents the effect of resonant particles on the wave modulations. Moreover, the parameter $\lambda$ gives rise to a damping effect. Indeed, let $E$ be the "energy" defined by

$$
E(u)=\int_{-\infty}^{+\infty}|u|^{2} d x
$$

*Received: February 8, 2010; accepted (in revised version): May 11, 2010. Communicated by Jack Xin.

${ }^{\dagger}$ Departamento de Matemática-CCN, Universidade Federal do Piauí, Campus Ministro Petronio Portela, SG-04 - Ininga, 64049-550, Teresina-PI, Brazil (mourapr@impa.br).

${ }^{\ddagger}$ IMECC-UNICAMP, Rua Sérgio Buarque de Holanda, 651, 13083-859, Campinas-SP, Brazil (apastor@ime.unicamp.br). 
A straightforward computation shows that if $u$ is a solution of (1.2) then

$$
\frac{d}{d t} E(u(t))=\lambda \int_{-\infty}^{+\infty}|u|^{2} \mathcal{H} \partial_{x}\left(|u|^{2}\right) d x
$$

Since the integral on the right-hand side of (1.4) is positive, we see that the energy either increases $(\lambda>0)$ or decreases $(\lambda<0)$ monotonically with time due to effect of resonant particles.

In many important situations, the parameter $\lambda$ turns out to be negative. For instance, if the waves are propagating in plasmas with isotropic Maxwellian distributed electrons and ions, the effect of resonant particles is weak when the Alfvén velocity is much larger than the ion sound velocity; in such a case,

$$
\lambda \simeq-\frac{1}{\sqrt{2 \pi}}\left(\frac{m_{\mathrm{e}}}{m_{\mathrm{i}}}\right)^{1 / 2} \frac{C_{\mathrm{s}}}{v_{\mathrm{A}}} \exp \left(-\frac{1}{2} \frac{v_{\mathrm{A}}^{2}}{v_{\mathrm{e}}^{2}}\right), \quad v_{\mathrm{A}} \gg C_{\mathrm{s}},
$$

where $m_{\mathrm{e}}, m_{\mathrm{i}}, C_{\mathrm{s}}, v_{\mathrm{A}}$, and $v_{\mathrm{e}}$ denote, respectively, the electron mass, the ion mass, the ion sound velocity, the Alfvén velocity, and the electron thermal velocity (see [21]). In general, it can be shown that a sufficient condition for $\lambda$ to be negative is that the velocity distributions decrease as functions of the parallel velocity component of the Alfvén velocity (see [21]).

Note that (1.2) becomes (1.1) in the case $\lambda=0$. Hence, for $|\lambda| \ll 1$ the nDNLS equation can also be viewed as a perturbation of the DNLS equation.

From the mathematical viewpoint, the DNLS equation has been extensively studied in recent years by several authors (see e.g. [1, 3]-[5, 9]-[14, 24, 28, 29], and references therein). On the other hand, a few works are available in the current literature for the nDNLS equation. As far as we know, the only works concerning well-posedness for $(1.2)$ are due to Rial $[26,27]$. In [26] the author studied the Cauchy problem (with $\lambda<0$ ); by using Kato's theory he proved the initial value problem for (1.2) is locally (in time) well-posed in the usual Sobolev spaces $H^{s}(\mathbb{R}), s>3 / 2$, and in the weighted spaces $H^{s}(\mathbb{R}) \cap L_{w}^{2}(\mathbb{R}), s>3 / 2$, for a suitable class of weights $w$. In [27], by introducing an artificial viscosity and then passing to the limit, the author proved the existence of weak solutions in $L^{2}(\mathbb{R})$ (also for $\lambda<0$ ).

The first purpose of this work is to improve the local well-posedness theory obtained in [26]. Throughout the paper, except in Section 4, by well-posedness we mean existence, uniqueness, persistence property, and continuous dependence upon the data. Moreover, by a solution we mean a solution in the sense of the associated integral equation. Our main result concerning well-posedness is the following (for the definitions of the space $X_{T}^{s}$ and $P_{0}$ see notations below).

TheOREm 1.1. Let $s>1 / 2$. Then there exists a $\delta>0$ such that for any $\phi \in H^{s}(\mathbb{R})$, with $\|\phi\|_{L^{2}}<\delta$, there exist a positive time $T=T\left(\|\phi\|_{H^{s}}\right)$ with $T\left(\|\phi\|_{H^{s}}\right) \rightarrow \infty$ as $\|\phi\|_{H^{s}} \rightarrow 0$, and a unique solution

$$
u \in C\left([-T, T] ; H^{s}(\mathbb{R})\right) \cap\left\{u ; e^{i \rho(u)} u \in X_{T}^{s}\right\}
$$

of (1.2) with initial data $\phi$, where $\rho(u)(x, t)=-\frac{1}{2} \lambda P_{0}\left(\int_{-\infty}^{x} \mathcal{H}\left(|u(y, t)|^{2}\right) d y\right)$. Furthermore, for any $T^{\prime} \in(0, T)$ there exists $\epsilon>0$ such that the flow-map data-solution is Lipschitz from $\left\{\psi \in H^{s}(\mathbb{R}) ;\|\widetilde{\phi}-\phi\|_{H^{s}}<\epsilon\right\}$ into $C\left(\left[-T^{\prime}, T^{\prime}\right] ; H^{s}(\mathbb{R})\right) \cap\left\{u ; e^{i \rho(u)} u \in X_{T^{\prime}}^{s}\right\}$.

As is well known, the main difficulty when one deals with equations containing derivatives in the nonlinear term, is to overcome the so-called loss of derivatives. In our 
case, the most difficult term is $\mathcal{H} P_{0}\left(|u|^{2}\right) \partial_{x} u$, because the Hilbert transform $\mathcal{H}$ is not bounded in $L^{1}$. Thus, inspired by the results for the DNLS equation, our approach to prove Theorem 1.1 is based on the Gauge transformation $v=e^{-i \frac{\lambda}{2} P_{0} \int_{-\infty}^{x} \mathcal{H}\left(|u(y, t)|^{2}\right) d y} u$, where $P_{0}$ is the projection operator into small frequencies.

It is worth noticing that the methods employed by Ozawa and Tsutsumi [24, 25], and by Takaoka [29] to study the Cauchy problem for a vast class of DNLS equation are not applicable in the case of the Cauchy problem associated with (1.2). In their approach, they basically depend on the Gauge transformation $v=e^{i \alpha \int_{-\infty}^{x}|u(y, t)|^{2} d y} u$ $(\alpha \in \mathbb{R}$ chosen conveniently). In fact, Ozawa [24] used that transformation to remove the derivative term from the nonlinearity and proved the well-posedness for a class of DNLS equations in the energy space $H^{1}$. Ozawa and Tsutsumi [25] proved the local well-posedness in $H^{\frac{1}{2}}$. On the other hand, Takaoka [29] applied the Fourier restriction norm method to show local well-posedness in $H^{s}, s \geq 1 / 2$, improving the results due to Ozawa and Tsutsumi. But via such a transformation the loss of derivatives still persists in our case under the form $v \mathcal{H}\left(\bar{v} \partial_{x} v\right)$ and, as observed in [29], the Fourier restriction norm method seems to be inapplicable to nonlinearities like that due to a logarithmic divergence involving the key trilinear estimate.

REMARK 1.2. It should be pointed out that the smallness condition in Theorem 1.1 is in agreement with the physical meaning of Equation (1.2), where the reductive perturbation expansion was applied presupposing that the amplitude $u$ is small and the length scale $x$ is large (see [8]).

REMARK 1.3. Note that Theorem 1.1 also includes the case $\lambda>0$, which was not studied in $[26,27]$.

Once Theorem 1.1 is proved, a natural question presents itself: can we show well-posedness in $H^{s}(\mathbb{R})$ for $s \leq 1 / 2$ ? By using a scaling argument we may have an insight on this question. To make this point clear, we note that if $u$ solves the Cauchy problem for $(1.2)$ with initial data $u_{0}$ then

$$
u_{\sigma}(x, t)=\sigma^{1 / 2} u\left(\sigma x, \sigma^{2} t\right)
$$

also solves the Cauchy problem for (1.2) with initial data $u_{\sigma}(x, 0)=\sigma^{1 / 2} u_{0}(\sigma x)$. Hence, the highest derivative that leaves invariant the $H^{s}(\mathbb{R})$-norm of $u_{\sigma}$ is $s=0$. Actually, a straightforward calculation reveals that

$$
\left\|u_{\sigma}(\cdot, 0)\right\|_{\dot{H}^{s}}=\sigma^{s}\left\|u_{0}\right\|_{\dot{H}^{s}},
$$

where $\dot{H}^{s}=\dot{H}^{s}(\mathbb{R})$ denotes the homogeneous Sobolev space of order $s$. This argument suggests that $L^{2}(\mathbb{R})$ is the critical space where we expect to prove a local well-posedness result. Meanwhile, contrary to this expectation, we prove that one cannot obtain local well-posedness in $H^{s}(\mathbb{R}), s<1 / 2$, in the sense that the flow-map data-solution is not $C^{3}$ at the origin. More precisely, we establish the following.

TheOREM 1.4. Let $s<1 / 2$. If the Cauchy problem for (1.2) is locally well-posed in $H^{s}(\mathbb{R})$, then the flow-map data-solution

$$
S(t): H^{s}(\mathbb{R}) \longrightarrow H^{s}(\mathbb{R}), \quad u_{0} \longmapsto u(t)
$$

is not $C^{3}$ at the origin.

This result is very similar to that obtained by Takaoka in [28], where the same result was proved for the DNLS Equation (1.1). The idea to prove Theorem 1.4 is to 
use the technique introduced by Bourgain [2], which consists in locating certain plane waves whose interactions behave badly in low regularity. We point out that results in the same direction were proved for the Korteweg-de Vries [30] and Benjamin-Ono [23] equations.

The second purpose of the paper is to investigate the (non)existence of solitarywave solutions. It is well known that the DNLS equation possesses a two-parameter family of solitary waves of the form (see [31])

$$
u_{\omega, c}(x, t)=e^{-i \omega t} e^{i \psi(x-c t)} \varphi(x-c t),
$$

where $\omega, c$ are real parameters satisfying $-c^{2}-4 \omega>0$, and $\psi, \varphi$ are smooth real-valued functions given by

$$
\left\{\begin{array}{l}
\varphi(x)=\left(d_{3}+d_{5} \cosh \left(d_{6} x\right)\right)^{-1 / 2}, \\
\psi^{\prime}(x)=\frac{c}{2}+\frac{3}{4} \varphi^{2}(x), \\
d_{3}=\frac{c}{2\left(-c^{2}-4 \omega\right)}, \quad d_{5}^{2}=\frac{-\omega}{\left(-c^{2}-4 \omega\right)^{2}}, \quad d_{6}^{2}=-c^{2}-4 \omega .
\end{array}\right.
$$

Therefore, since the nDNLS equation can be viewed as a perturbation of the DNLS equation, one could expect, at least for sufficiently small $\lambda$, the existence of solitary waves for (1.2) of the form (1.8). As a matter of fact, this question was addressed in [32] using both numerical simulations and the soliton perturbation method. But, as was observed by the authors (in their summary), it is not clear if the methods could prove the existence of solitary waves for (1.2) because the non-soliton part should always appear in the eigenvalue problem, and as a result all solitons eventually disappear.

Here, we look for solitary-wave solutions of the form

$$
u_{\omega, c}(x, t)=e^{-i \omega t} \zeta(x-c t),
$$

where $\zeta$ is a complex-valued function, and $\omega$ and $c$ are real parameters. By assuming that $\zeta$ has finite energy, we prove that, for any $\lambda \neq 0$, solitary waves of the form (1.10) indeed do not exist. More precisely, we prove the following:

TheOREM 1.5. Let $\zeta \in L^{2}(\mathbb{R})$ such that

$$
\int_{-\infty}^{+\infty}|\zeta|^{2} \mathcal{H} \partial_{x}\left(|\zeta|^{2}\right) d x<\infty
$$

Then, for any $\lambda \neq 0,(1.2)$ does not admit any nontrivial solitary-wave solutions of the form (1.10), for any $\omega, c \in \mathbb{R}$.

The proof of Theorem 1.5 is standard, and it follows essentially from the lack of energy conservation.

It should be pointed out that the orbital stability of the solitary waves (1.9) was addressed in [3] and [11]. Moreover, by using such solitary waves Biagioni and Linares [1] proved the ill-posedness in $H^{s}(\mathbb{R}), s<1 / 2$, for the Cauchy problem associated with the DNLS equation, in the sense that the flow-map data-solution is not uniformly continuous. 
Besides this introduction, this work is organized as follows. In Section 2 we list the smoothing effects for the linear Schrödinger equation and the fractional vector-valued Leibniz rule estimates to be used throughout the paper. In Section 3 we derive our Gauge transformation and give the proof of Theorem 1.1. In Section 4 we prove the ill-posedness result. Finally, in Section 5, we prove the nonexistence of solitary-wave solutions.

Notation. Given any positive constants $C, D$, by $C \lesssim D$ we mean that there exists a constant $c>0$ such that $C \leq c D$. By $C \sim D$ we mean $C \lesssim D$ and $D \lesssim C$. Given two operators $A$ and $B$, we denote by $[A, B]=A B-B A$ the commutator between $A$ and $B$. By $\mathcal{F}\{u\}$ or $\widehat{u}$ we denote the Fourier transform of $u$ with respect to the space variable $x$, while $\mathcal{F}^{-1}\{u\}$ or $\breve{u}$ denote its inverse Fourier transform. $L^{p}$-norms are written as $\|\cdot\|_{L_{x}^{p}}$ or $\|\cdot\|_{L^{p}}$ if no confusion is caused. For $1 \leq p, q<\infty$ and $f: \mathbb{R} \times[0, T] \rightarrow \mathbb{R}$, we define

$$
\|f\|_{L_{x}^{p} L_{T}^{q}}=\left(\int_{-\infty}^{\infty}\left(\int_{0}^{T}|f(x, t)|^{q} d t\right)^{p / q} d x\right)^{1 / p} .
$$

$\|f\|_{L_{T}^{q} L_{x}^{p}}$ is similarly defined, and when $p=\infty$ or $q=\infty,\|f\|_{L_{x}^{p} L_{T}^{q}}$ is defined in the natural form. When $p=q$, we write $\|f\|_{L_{x, T}^{p}}$ instead of $\|f\|_{L_{x}^{p} L_{T}^{q}}$.

$\mathcal{S}(\mathbb{R})$ represents the Schwartz space. $J^{s}$ will be the Bessel potential, $J^{s}=(1-$ $\left.\partial_{x}^{2}\right)^{s / 2}=\mathcal{F}^{-1}\left\{\left(1+|\cdot|^{2}\right)^{s / 2}\right\}, D_{x}^{s}$ denotes the Riesz potential $D_{x}^{s}=\left(-\partial_{x}^{2}\right)^{s / 2}=\mathcal{F}^{-1}\{\mid \cdot$ $\left.\left.\right|^{s}\right\}$, and $\widetilde{D}_{x}^{s}=\mathcal{H} D_{x}^{s}$. The space $H^{s}(\mathbb{R})$ is the usual Sobolev space with norm $\|\cdot\|_{H^{s}}:=$ $\left\|J^{s} \cdot\right\|_{L^{2}}$.

Let $\psi$ be an even $C_{0}^{\infty}$ function such that $\psi(\xi)=1$ for all $|\xi| \leq 1$ and $\psi(\xi)=0$ for $|\xi| \geq 2$. Set $\varphi(\xi)=\psi(\xi)-\psi(2 \xi)$. For any dyadic number $N=2^{j}, j \in \mathbb{Z}_{\geq 0}$, write $\varphi_{N}(\xi)=\varphi(\xi / N)$, and define $\varphi_{0}$ by $\varphi_{0}(\xi)=1-\sum_{N=2^{j} \geq 1} \varphi_{N}(\xi)$. Let $P_{N}$ be the convolution operators $P_{N} f=\check{\varphi}_{N} * f$ and $P_{0} f=\check{\varphi}_{0} * f$.

We denote by $P_{+} f=\mathcal{F}^{-1}\left\{\chi_{[0,+\infty)}(\cdot) \widehat{f}(\cdot)\right\}$ and $P_{-} f=\mathcal{F}^{-1}\left\{\chi_{(-\infty, 0]}(\cdot) \widehat{f}(\cdot)\right\}$ the projection in positive and negative frequencies of $f$, respectively. We finally define $P_{+h i} f=P_{+}\left(\left(1-P_{0}\right) f\right)$ and $P_{-h i} f=P_{-}\left(\left(1-P_{0}\right) f\right)$. It is well known that $P_{-h i}$ and $P_{+h i}$ are continuous operators on $L_{x}^{p} L_{T}^{q}$ for any $1 \leq p, q \leq \infty$.

We now exhibit the resolution space $X_{T}^{s}$. Given $s>1 / 2$ and $0<T \leq 1$, we define

$$
X_{T}^{s}=\left\{u \in C\left([-T, T] ; H^{s}(\mathbb{R})\right) ;\|u\|_{X_{T}^{s}}<\infty\right\},
$$

where

$$
\begin{gathered}
\|u\|_{X_{T}^{s}}:=\max \left\{\|u\|_{L_{T}^{\infty} H_{x}^{s}},\left\|D_{x}^{s+\frac{1}{2}} u\right\|_{L_{x}^{\infty} L_{T}^{2}},\|u\|_{L_{x}^{2} L_{T}^{\infty}},\left\|D_{x}^{s} u\right\|_{L_{x, T}^{6}},\left\|D_{x}^{\frac{1}{2}} u\right\|_{L_{T, x}^{6}},\right. \\
\left.\left\|D_{x}^{s+\frac{1}{10}} u\right\|_{L_{x}^{5} L_{T}^{\frac{10}{3}}},\left\|D_{x}^{\frac{9}{10}} u\right\|_{L_{x}^{20} L_{T}^{\frac{20}{9}}}\right\} .
\end{gathered}
$$

\section{Linear estimates and auxiliary Lemmas}

Solutions of the IVP associated with the linear Schrödinger equation

$$
\left\{\begin{array}{l}
\partial_{t} u-i \partial_{x}^{2} u=0, x, t \in \mathbb{R} \\
u(x, 0)=\phi(x)
\end{array}\right.
$$

are described by the unitary group $\left\{e^{i t \partial_{x}^{2}}\right\}_{t=-\infty}^{\infty}$, which from now on will be symbolized by $\{U(t)\}_{t=-\infty}^{\infty}$. 
Definition 2.1. We say that a triplet $(\alpha, p, q) \in \mathbb{R} \times[2, \infty]^{2}$ is

(i) 1-admissible if

$$
(\alpha, p, q)=\left(\frac{1}{2}, \infty, 2\right) \text { or } p \in[4, \infty), q \in[2, \infty], \frac{2}{p}+\frac{1}{q} \leq \frac{1}{2}, \alpha=\frac{1}{p}+\frac{2}{q}-\frac{1}{2} ;
$$

(ii) 2-admissible if $2<p, q<\infty, \quad \frac{1}{p}+\frac{1}{q} \leq \frac{1}{2}, \quad \alpha=\frac{1}{p}+\frac{3}{q}-1$. If moreover $4 \leq$ $p<\infty$, we say that $(\alpha, p, q)$ is $2^{*}$-admissible.

We first list the smoothing effects and Strichartz estimates obtained by Molinet and Ribaud in [22], using previous results of Kenig, Ponce, and Vega [15, 17].

Lemma 2.2. Let $(\alpha, p, q) \in \mathbb{R} \times[2, \infty]^{2}$ and $0<T<1$.

(i) If $(\alpha, p, q)$ is 1-admissible, then

$$
\left\|D_{x}^{\alpha} U(t) \phi\right\|_{L_{x}^{p} L_{T}^{q}} \lesssim\|\phi\|_{L^{2}}, \quad \forall \phi \in \mathcal{S}(\mathbb{R}) .
$$

(ii) If $(\alpha, p, q)$ is 2-admissible, then

$$
\left\|J_{x}^{\alpha} U(t) \phi\right\|_{L_{x}^{p} L_{T}^{q}} \lesssim\|\phi\|_{L^{2}}, \quad \forall \phi \in \mathcal{S}(\mathbb{R}) .
$$

(iii) If $(\alpha, p, q)$ is $2^{*}$-admissible, then

$$
\left\|D_{x}^{\alpha} U(t) \phi\right\|_{L_{x}^{p} L_{T}^{q}} \lesssim T^{\frac{1}{4}-\frac{1}{2 q}}\|\phi\|_{L^{2}}, \quad \forall \phi \in \mathcal{S}(\mathbb{R}) .
$$

The following maximal function estimate will be essential.

Lemma 2.3. For any $s>1 / 2$ and $0<T \leq 1$, we have

$$
\|U(t) \phi\|_{L_{x}^{2} L_{T}^{\infty}} \lesssim\|\phi\|_{H^{s}} .
$$

Proof. Cf. [16, Theorem 3.1].

Making use of the so-called Christ and Kiselev Lemma [6], Molinet and Ribaud [22] deduced the following retarded estimates from the above nonretarded ones.

Lemma 2.4. Let $\left(\alpha_{1}, \alpha_{2}\right) \in \mathbb{R}^{2},\left(r_{1}, r_{2}\right) \in \mathbb{R}_{+}^{2}$ and $1 \leq p_{1}, q_{1}, p_{2}, q_{2} \leq \infty$ be such that, given $\phi \in \mathcal{S}(\mathbb{R})$,

$$
\begin{gathered}
\left\|D_{x}^{\alpha_{1}} U(t) \phi\right\|_{L_{x}^{p_{1}} L_{T}^{q_{1}}} \lesssim T^{r_{1}}\|\phi\|_{L^{2}}, \\
\left\|D_{x}^{\alpha_{2}} U(t) \phi\right\|_{L_{x}^{p_{2}} L_{T}^{q_{2}}} \lesssim T^{r_{2}}\|\phi\|_{L^{2} .} .
\end{gathered}
$$

Then for all $F \in \mathcal{S}(\mathbb{R})$,

$$
\begin{gathered}
\left\|D_{x}^{\alpha_{2}} \int_{0}^{t} U(t-\tau) F(\cdot, \tau) d \tau\right\|_{L_{T}^{\infty} L_{x}^{2}} \lesssim T^{r_{2}}\|F\|_{L_{x}^{\tilde{p}_{2}} L_{T}^{\tilde{q}_{2}}}, \\
\left\|D_{x}^{\alpha_{1}+\alpha_{2}} \int_{0}^{t} U(t-\tau) F(\cdot, \tau) d \tau\right\|_{L_{x}^{p_{1}} L_{T}^{q_{1}}} \lesssim T^{r_{1}+r_{2}}\|F\|_{L_{x}^{\tilde{p}_{2}} L_{T}^{\tilde{q}_{2}}},
\end{gathered}
$$

provided

$$
\min \left(p_{1}, q_{1}\right)>\max \left(\tilde{p}_{2}, \tilde{q}_{2}\right) \text { or }\left(q_{1}=\infty \text { and } \tilde{p}_{2}, \tilde{q}_{2}<\infty\right),
$$


where $\tilde{p}_{2}, \tilde{q}_{2}$ are defined by $\frac{1}{\tilde{p}_{2}}=1-\frac{1}{p_{2}}$ and $\frac{1}{\tilde{q}_{2}}=1-\frac{1}{q_{2}}$.

As a consequence of Lemmas 2.2 and 2.3, one has the following result which guarantees that $U(t) \phi \in X_{T}^{s}$.

Lemma 2.5. Let $s>1 / 2$ and $T \in(0,1)$. Then for all $\phi \in H^{s}$,

$$
\|U(t) \phi\|_{X_{T}^{s}} \lesssim\|\phi\|_{H^{s}} \text { and }\left\|U(t) P_{0} \phi\right\|_{X_{T}^{s}} \lesssim\|\phi\|_{L^{2}} .
$$

Proof. Actually, (2.11) is an immediate consequence of Lemma 2.2, Lemma 2.3 and Bernstein's inequality. It is enough to observe that $\left(\frac{1}{2}, \infty, 2\right),(0,6,6)$ are 1 admissible, and $\left(\frac{1}{10}, 5, \frac{10}{3}\right)$ and $\left(\frac{2}{5}, 20, \frac{20}{9}\right)$ are $2^{*}$-admissible.

Finally, we recall some vector-valued Leibniz rules for fractional derivatives, which are fundamental for reaching our goal.

LEMmA 2.6. Let $\alpha \in(0,1), \quad \alpha_{1}, \alpha_{2} \in[0, \alpha]$ such that $\alpha=\alpha_{1}+\alpha_{2}$. Then if $p, p_{1}, p_{2}, q, q_{1}, q_{2} \in(1, \infty)$, with $\frac{1}{p}=\frac{1}{p_{1}}+\frac{1}{p_{2}}$ and $\frac{1}{q}=\frac{1}{q_{1}}+\frac{1}{q_{2}}$, we have

(i) $\left\|D_{x}^{\alpha}(f g)-f D_{x}^{\alpha} g-g D_{x}^{\alpha} f\right\|_{L_{x}^{p} L_{T}^{q}} \leq c\left\|D_{x}^{\alpha_{1}} f\right\|_{L_{x}^{p_{1}} L_{T}^{q_{1}}}\left\|D_{x}^{\alpha_{2}} g\right\|_{L_{x}^{p_{2}} L_{T}^{q_{2}}}$.

Moreover, if $\alpha_{1}=0$, (i) still holds with $q_{1}=\infty$.

(ii) $\left\|D_{x}^{\beta}\left(\left[D_{x}^{\alpha}, f\right] g\right)\right\|_{L_{x}^{p} L_{T}^{q}} \lesssim\|g\|_{L_{x}^{p_{1}} L_{T}^{q_{1}}}\left\|D_{x}^{\alpha+\beta} f\right\|_{L_{x}^{p_{2}} L_{T}^{q_{2}}}$, where $0 \leq \beta<1-\alpha$. Moreover, for $\beta>0$ the value $q_{1}=\infty$ is allowed.

(iii) $\left\|D_{x}^{\alpha}(f g)-f D_{x}^{\alpha} g-g D_{x}^{\alpha} f\right\|_{L_{x}^{1} L_{T}^{2}} \leq c\left\|D_{x}^{\alpha_{1}} f\right\|_{L_{x}^{p_{1}} L_{T}^{q_{1}}}\left\|D_{x}^{\alpha_{2}} g\right\|_{L_{x}^{p_{2}} L_{T}^{q_{2}}}$, where $1=\frac{1}{p_{1}}+\frac{1}{p_{2}}, \frac{1}{2}=\frac{1}{q_{1}}+\frac{1}{q_{2}}$ and $p_{1}, p_{2}, q_{1}, q_{2} \in(1, \infty)$.

(iv) $\left\|D_{x}^{\alpha} P_{ \pm}\left(f P_{\mp} D_{x}^{\beta} g\right)\right\|_{L_{x}^{p} L_{T}^{q}} \leq c\left\|D_{x}^{\gamma_{1}} f\right\|_{L_{x}^{p_{1}} L_{T}^{q_{1}}}\left\|D_{x}^{\gamma_{2}} g\right\|_{L_{x}^{p_{2}} L_{T}^{q_{2}}}$, with $\gamma_{1} \geq \alpha$ and $\gamma_{1}+\gamma_{2}=\alpha+\beta$.

(v) $\left\|D_{x}^{\alpha}(f g)-f D_{x}^{\alpha} g-g D_{x}^{\alpha} f\right\|_{L_{x}^{p}} \lesssim\|g\|_{L_{x}^{\infty}}\left\|D_{x}^{\alpha} f\right\|_{L_{x}^{p} \text {. }}$

The lemma is still valid with $\widetilde{D}_{x}^{\alpha}$ in place of $D_{x}^{\alpha}$.

Proof. Parts (i), (iii), and (v) were proved by Kenig, Ponce, and Vega[18] (Theorems A.8, A13, and A12, respectively). Parts (ii) and (iv) (this last one for the case $P_{+}\left(f P_{-} g\right)$ ) were established by Molinet and Ribaud [22] (Lemmas 3.4 and 3.5, respectively).

\section{Proof of Theorem 1.1}

3.1. Gauge transformation. Let $u$ be a solution to (1.2) with sufficient regularity in space-time and integrability in space. Inspired by [25], we perform the following change of dependent variable,

$$
v(x, t)=e^{i \rho(u)} u(x, t), \text { with } \rho=\rho(u(x, t))=\beta \lambda P_{0}\left(\int_{-\infty}^{x} \mathcal{H}\left(|u(y, t)|^{2}\right) d y\right),
$$

where $\beta$ is a real number to be chosen later. Then a direct calculation shows that $v$ satisfies

$$
\partial_{t} v-i \partial_{x}^{2} v=i e^{i \rho} u\left\{\partial_{t} \rho-i \partial_{x}^{2} \rho+\left(\partial_{x} \rho\right)^{2}\right\}+e^{i \rho}\left(\partial_{t} u-i \partial_{x}^{2} u\right)+2 \partial_{x} \rho e^{i \rho} \partial_{x} u .
$$

By (1.2) and (3.1), we see that

$$
\partial_{t} \rho=\beta \lambda \mathcal{H} P_{0}\left(\int_{-\infty}^{x}\left(\partial_{t} u \bar{u}+u \partial_{t} \bar{u}\right) d y\right)=\beta \lambda \mathcal{H} P_{0}(I)
$$


where

$$
\begin{aligned}
I= & \int_{-\infty}^{x}\left(i \partial_{y}^{2} u \bar{u}-i u \partial_{y}^{2} \bar{u}\right) d y+\int_{-\infty}^{x} \partial_{y}\left(|u|^{2} u\right) \bar{u} d y+\int_{-\infty}^{x} \partial_{y}\left(|u|^{2} \bar{u}\right) u d y \\
& +\lambda \int_{-\infty}^{x} \partial_{y}\left(\mathcal{H}\left(|u|^{2}\right) u\right) \bar{u} d y+\lambda \int_{-\infty}^{x} \partial_{y}\left(\mathcal{H}\left(|u|^{2}\right) \bar{u}\right) u d y \\
= & i\left(\partial_{x} u \bar{u}-u \partial_{x} \bar{u}\right)+\frac{3}{2}|u|^{4}+2 \lambda \int_{-\infty}^{x} \mathcal{H} \partial_{y}\left(|u|^{2}\right)|u|^{2} d y+\lambda \int_{-\infty}^{x} \mathcal{H}\left(|u|^{2}\right) \partial_{y}\left(|u|^{2}\right) d y \\
= & i\left(\partial_{x} u \bar{u}-u \partial_{x} \bar{u}\right)+\frac{3}{2}|u|^{4}+\lambda \mathcal{H}\left(|u|^{2}\right)|u|^{2}+\lambda \int_{-\infty}^{x} \mathcal{H} \partial_{y}\left(|u|^{2}\right)|u|^{2} d y
\end{aligned}
$$

Since $|u|=|v|$ and $e^{i \rho} \partial_{x} u=\partial_{x} v-i \beta \lambda v \mathcal{H} P_{0}\left(|v|^{2}\right)$, we have

$$
\mathcal{H} P_{0}\left(u \partial_{x} \bar{u}\right)=\mathcal{H} P_{0}\left(v \partial_{x} \bar{v}\right)+i \beta \lambda \mathcal{H} P_{0}\left(|v|^{2} \mathcal{H} P_{0}\left(|v|^{2}\right)\right) .
$$

Then, from (3.1) and (3.3)-(3.5), we deduce that

$$
\begin{aligned}
\partial_{t} \rho-i \partial_{x}^{2} \rho+\left(\partial_{x} \rho\right)^{2}= & -2 i \beta \lambda \mathcal{H} P_{0}\left(v \partial_{x} \bar{v}\right)+2 \beta^{2} \lambda^{2} \mathcal{H} P_{0}\left(|v|^{2} \mathcal{H} P_{0}\left(|v|^{2}\right)+\frac{3 \beta \lambda}{2} \mathcal{H} P_{0}\left(|v|^{4}\right)\right. \\
& +\beta \lambda^{2} \mathcal{H} P_{0}\left(|v|^{2} \mathcal{H}\left(|v|^{2}\right)\right)+\beta \lambda^{2} \mathcal{H} P_{0}\left(\int_{-\infty}^{x} \mathcal{H} \partial_{y}\left(|v|^{2}\right)|v|^{2} d y\right) \\
& +\beta^{2} \lambda^{2}\left(\mathcal{H} P_{0}\left(|v|^{2}\right)\right)^{2}
\end{aligned}
$$

Thus $v$ satisfies the equation

$$
\begin{aligned}
\partial_{t} v-i \partial_{x}^{2} v= & 2 \beta \lambda v \mathcal{H} P_{0}\left(v \partial_{x} \bar{v}\right)+2 i \beta^{2} \lambda^{2} v \mathcal{H} P_{0}\left(|v|^{2} \mathcal{H} P_{0}\left(|v|^{2}\right)\right)+\frac{3 i}{2} \beta \lambda v \mathcal{H} P_{0}\left(|v|^{4}\right) \\
& +i \beta \lambda^{2} v \mathcal{H} P_{0}\left(|v|^{2} \mathcal{H}\left(|v|^{2}\right)\right)+i \beta \lambda^{2} v \mathcal{H} P_{0}\left(\int_{-\infty}^{x} \mathcal{H} \partial_{y}\left(|v|^{2}\right)|v|^{2} d y\right)+\partial_{x}\left(|v|^{2} v\right) \\
& -i \beta \lambda v|v|^{2} \mathcal{H} P_{0}\left(|v|^{2}\right)+\lambda v \mathcal{H} \partial_{x}\left(|v|^{2}\right)+\lambda \mathcal{H} P_{h i}\left(|v|^{2}\right) \partial_{x} v \\
& -i \beta \lambda^{2} v \mathcal{H} P_{0}\left(|v|^{2}\right) \mathcal{H}\left(|v|^{2}\right)+(2 \beta+1) \lambda \mathcal{H} P_{0}\left(|v|^{2}\right) \partial_{x} v \\
& -2 i \beta^{2} \lambda^{2} v\left(\mathcal{H} P_{0}\left(|v|^{2}\right)\right)^{2} .
\end{aligned}
$$

As we have already said in the introduction, the main difficulty is to handle the term $\mathcal{H} P_{0}\left(|v|^{2}\right) \partial_{x} v$. To overcome this difficulty we choose $\beta=-1 / 2$. Taking that value for $\beta$ in (3.6), we obtain the following equation:

$$
\begin{aligned}
\partial_{t} v-i \partial_{x}^{2} v= & \partial_{x}\left(|v|^{2} v\right)+\lambda \mathcal{H} P_{h i}\left(|v|^{2}\right) \partial_{x} v+\lambda v \mathcal{H} \partial_{x}\left(|v|^{2}\right)-\lambda v \mathcal{H} P_{0}\left(v \partial_{x} \bar{v}\right) \\
& -i \frac{\lambda^{2}}{2} v \mathcal{H} P_{0}\left(\int_{-\infty}^{x} \mathcal{H} \partial_{y}\left(|v|^{2}\right)|v|^{2} d y\right)+i \frac{\lambda}{2} v|v|^{2} \mathcal{H} P_{0}\left(|v|^{2}\right)-i \frac{3 \lambda}{4} v \mathcal{H} P_{0}\left(|v|^{4}\right) \\
& +i \frac{\lambda^{2}}{2} v \mathcal{H} P_{0}\left(|v|^{2} \mathcal{H} P_{0}\left(|v|^{2}\right)\right)-i \frac{\lambda^{2}}{2} v \mathcal{H} P_{0}\left(\mathcal{H}\left(|v|^{2}\right)|v|^{2}\right) \\
& +i \frac{\lambda^{2}}{2} v \mathcal{H} P_{0}\left(|v|^{2}\right) \mathcal{H}\left(|v|^{2}\right)-i \frac{\lambda^{2}}{2} v\left(\mathcal{H} P_{0}\left(|v|^{2}\right)\right)^{2}=: \sum_{j=1}^{11} c_{j} F_{j}(v)
\end{aligned}
$$

where $F_{j}=F_{j}(v)$ are numbered according to their position in (3.7).

Conversely, a similar calculation reveals that if $v$ satisfies $(3.7)$, then $u(x, t)=$ $e^{-i \rho(v)} v(x, t)$ satisfies (1.2), where $\rho$ is defined in (3.1) with $\beta=-1 / 2$. 
3.2. Local well-posedness for the Gauge transformed equation. The main goal here is to establish local well-posedness for the Cauchy problem associated with (3.7). Hence we consider the IVP

$$
\left\{\begin{array}{l}
\partial_{t} v-i \partial_{x}^{2} v=\sum_{j=1}^{11} c_{j} F_{j}, x, t \in \mathbb{R}, \\
v(x, 0)=\psi(x) .
\end{array}\right.
$$

The idea to prove the solvability of (3.8) is to use the Banach contraction principle. More precisely, we prove the following.

Proposition 3.1. Let $s>1 / 2$. Then there exists a $\delta>0$ such that for any $\psi \in$ $H^{s}(\mathbb{R})$, with $\|\psi\|_{H^{s}}<\delta$, there exist a positive time $T=T\left(\|\psi\|_{H^{s}}\right)$ with $T\left(\|\psi\|_{H^{s}}\right) \rightarrow \infty$ as $\|\psi\|_{H^{s}} \rightarrow 0$, a space $X_{T}^{s}$ such that $X_{s}^{T} \hookrightarrow C\left([-T, T] ; H^{s}(\mathbb{R})\right)$, and a unique solution $u$ to the Cauchy problem (3.8) in $X_{T}^{s}$. Furthermore, for any $T^{\prime} \in(0, T)$ there exists $\epsilon>0$ such that the flow-map data-solution is Lipschitz from $\left\{\widetilde{\psi} \in H^{s}(\mathbb{R}) ;\|\widetilde{\psi}-\psi\|_{H^{s}}<\epsilon\right\}$ into $X_{T^{\prime}}^{s}$.

Proof. Let $s>1 / 2$ and $\psi \in H^{s}$ such that $\|\psi\|_{L^{2}}<\delta$, where $\delta$ will be chosen later. We look for a solution of the integral equation corresponding to (3.8), namely,

$$
v(t)=U(t) \psi+\sum_{j=1}^{11} c_{j} \int_{0}^{t} U(t-\tau) F_{j}(\tau) d \tau .
$$

Consider the map

$$
\Phi(v)(t)=U(t) \psi+\sum_{j=1}^{11} c_{j} \int_{0}^{t} U(t-\tau) F_{j}(\tau) d \tau .
$$

We shall prove that there exist $a=a\left(\|\psi\|_{H^{s}}\right)>0$ and $T(0<T \leq 1)$ such that, if $v \in X_{T}^{s}(a):=\left\{u \in X_{T}^{s} ;\|u\|_{X_{T}^{s}} \leq a\right\}$, then $\Phi(v) \in X_{T}^{s}(a)$ and $\Phi: X_{T}^{s}(a) \rightarrow X_{T}^{s}(a)$ is a contraction. Without loss of generality, we restrict ourselves to the most interesting case $1 / 2<s<9 / 10$. The case $s \geq 9 / 10$ can be handled in a similar fashion.

First we note that by Lemmas 2.3 and 2.5 we have, for $0<T \leq 1$, that

$$
\|U(t) \psi\|_{X_{T}^{s}} \lesssim\|\psi\|_{H^{s}} .
$$

To make the approach as easy as possible, we shall consider each term of (3.10) separately. At this stage we may take $\lambda=1$ in (3.7) without loss of generality. Our first strategy is to split the nonlinearities containing derivatives in the following way:

$$
\begin{aligned}
& F_{1}=\partial_{x}\left(|v|^{2} v\right)=2\left[D_{x}^{\frac{1}{2}},|v|^{2}\right] \widetilde{D}_{x}^{\frac{1}{2}} v+\left[D_{x}^{\frac{1}{2}}, v^{2}\right] \widetilde{D}_{x}^{\frac{1}{2}} \bar{v}-2 D_{x}^{\frac{1}{2}}\left(|v|^{2} \widetilde{D}_{x}^{\frac{1}{2}} v\right)-D_{x}^{\frac{1}{2}}\left(v^{2} \widetilde{D}_{x}^{\frac{1}{2}} \bar{v}\right) \\
& =\sum_{l=1}^{4} F_{1, l}, \\
& F_{2}=\left[D_{x}^{\frac{1}{2}}, \mathcal{H} P_{h i}\left(|v|^{2}\right)\right] \widetilde{D}_{x}^{\frac{1}{2}} v-D_{x}^{\frac{1}{2}}\left(\mathcal{H} P_{h i}\left(|v|^{2}\right) \widetilde{D}_{x}^{\frac{1}{2}} v\right)=\sum_{l=1}^{2} F_{2, l}, \\
& F_{3}=v \mathcal{H} \partial_{x}\left(|v|^{2}\right)= \\
& \quad P_{0}\left(v D_{x}\left(|v|^{2}\right)\right)+i P_{+h i}\left(v P_{-} \partial_{x}\left(|v|^{2}\right)\right)-i P_{+h i}\left(v P_{+} \partial_{x}\left(|v|^{2}\right)\right) \\
& \quad+i P_{-h i}\left(v P_{-} \partial_{x}\left(|v|^{2}\right)\right)-i P_{-h i}\left(v P_{+} \partial_{x}\left(|v|^{2}\right)\right)
\end{aligned}
$$




$$
\begin{aligned}
= & P_{0}\left(v D_{x}\left(|v|^{2}\right)\right)+2 i P_{+h i}\left(v P_{-} \partial_{x}\left(|v|^{2}\right)\right)-2 i P_{-h i}\left(v P_{+} \partial_{x}\left(|v|^{2}\right)\right) \\
& +i P_{+h i} D_{x}^{\frac{1}{2}}\left(|v|^{2} \widetilde{D}_{x}^{\frac{1}{2}} v\right)-i P_{+h i}\left(\left[D_{x}^{\frac{1}{2}},|v|^{2}\right] \widetilde{D}_{x}^{\frac{1}{2}} v\right)+i P_{+h i} D_{x}^{\frac{1}{2}}\left(v^{2} \widetilde{D}_{x}^{\frac{1}{2}} \bar{v}\right) \\
& -i P_{+h i}\left(\left[D_{x}^{\frac{1}{2}}, v^{2}\right] \widetilde{D}_{x}^{\frac{1}{2}} \bar{v}\right)+i P_{-h i}\left(\left[D_{x}^{\frac{1}{2}},|v|^{2}\right] \widetilde{D}_{x}^{\frac{1}{2}} v\right)-i P_{-h i} D_{x}^{\frac{1}{2}}\left(|v|^{2} \widetilde{D}_{x}^{\frac{1}{2}} v\right) \\
& +i P_{-h i}\left(\left[D_{x}^{\frac{1}{2}}, v^{2}\right] \widetilde{D}_{x}^{\frac{1}{2}} \bar{v}\right)-i P_{-h i} D_{x}^{\frac{1}{2}}\left(v^{2} \widetilde{D}_{x}^{\frac{1}{2}} \bar{v}\right)=\sum_{l=1}^{11} F_{3, l},
\end{aligned}
$$

and

$$
F_{4}=v \mathcal{H} P_{0}\left(v \partial_{x} \bar{v}\right)=v \mathcal{H} P_{0}\left(\left[D_{x}^{\frac{1}{2}}, v\right] \widetilde{D}_{x}^{\frac{1}{2}} \bar{v}\right)-v P_{0} \widetilde{D}_{x}^{\frac{1}{2}}\left(v \widetilde{D}_{x}^{\frac{1}{2}} \bar{v}\right)=\sum_{l=1}^{2} F_{4, l}
$$

Note that, unlike the other terms, $v \mathcal{H} \partial_{x}\left(|v|^{2}\right)$ was first divided in low and high frequencies parts. The reason for this is that to obtain the required estimates, we shall need to work in $L_{x}^{1} L_{T}^{2}$ and, as we know, $P_{ \pm}$are not bounded in such a space.

The estimate for the term $F_{5}=v \mathcal{H} P_{0}\left(\int_{-\infty}^{x} \mathcal{H} \partial_{y}\left(|u|^{2}\right)|u|^{2} d y\right)$, being slightly different, will be treated last.

Let

$$
E_{j}=\left\|\int_{0}^{t} U(t-\tau) F_{j}(\tau) d \tau\right\|_{X_{T}^{s}} \quad \text { and } \quad E_{j, l}=\left\|\int_{0}^{t} U(t-\tau) F_{j, l}(\tau) d \tau\right\|_{X_{T}^{s}} .
$$

Let $w$ denote either $v^{2},|v|^{2}$ or $\mathcal{H} P_{h i}\left(|v|^{2}\right)$, and let $\nu$ represent either $v$ or $\bar{v}$. Since $\left(\frac{2}{5}, 20, \frac{20}{9}\right)$ is $2^{*}$-admissible, $\left\|P_{h i}(\cdot)\right\|_{H^{s}} \sim\left\|P_{h i}(\cdot)\right\|_{H^{s}}$ and $P_{h i}, P_{ \pm h i}, \mathcal{H} P_{h i}$ are bounded in $L_{x}^{p} L_{T}^{q}, 1 \leq p, q \leq \infty$, and by Lemma 2.5, Lemma 2.5(i)-(ii), Minkowski and Hölder's inequalities, we obtain

$$
\begin{aligned}
& E_{1,1}+E_{1,2}+E_{2,1}+E_{3,5}+E_{3,7}+E_{3,8}+E_{3,10} \\
\lesssim & T^{\frac{1}{2}}\left\|\left[D_{x}^{\frac{1}{2}}, w\right] \widetilde{D}_{x}^{\frac{1}{2}} \nu\right\|_{L_{x, T}^{2}}+T^{\frac{1}{40}}\left\|D_{x}^{s-\frac{2}{5}}\left[D_{x}^{\frac{1}{2}}, w\right] \widetilde{D}_{x}^{\frac{1}{2}} \nu\right\|_{L_{x}^{\frac{20}{19}} L_{T}^{\frac{20}{11}}} \\
\lesssim & T^{\frac{1}{2}}\left\|D_{x}^{\frac{1}{2}} w\right\|_{L_{x, T}^{3}}\left\|D_{x}^{\frac{1}{2}} \nu\right\|_{L_{x, T}^{6}}+T^{\frac{1}{40}}\left\|D_{x}^{\frac{1}{2}} v\right\|_{L_{x, T}^{4}}\left\|D_{x}^{s+\frac{1}{10}} w\right\|_{L_{x}^{\frac{10}{7}} L_{T}^{\frac{10}{3}}} \\
\lesssim & T^{\frac{1}{2}}\left\|D_{x}^{\frac{1}{2}} v\right\|_{L_{x, T}^{6}}^{3}+T^{\frac{3}{20}}\|v\|_{L_{T}^{\infty} H^{\frac{1}{2}}}^{\frac{1}{4}}\left\|D_{x}^{\frac{1}{2}} v\right\|_{L_{x, T}^{6}}^{\frac{3}{4}}\|v\|_{L_{x}^{2} L_{T}^{\infty}}\left\|D_{x}^{s+\frac{1}{10}} v\right\|_{L_{x}^{5} L_{T}^{\frac{10}{3}}} .
\end{aligned}
$$

Because $P_{h i}, P_{ \pm h i}, \mathcal{H} P_{h i}$ are bounded in $L_{x}^{p} L_{T}^{q}, 1 \leq p, q \leq \infty$, by Lemma 2.4 we have

$$
\begin{aligned}
& E_{1,3}+E_{1,4}+E_{2,2}+E_{3,4}+E_{3,6}+E_{3,9}+E_{3,11} \\
\lesssim & T^{\frac{1}{2}}\left\|P_{0} D_{x}^{\frac{1}{2}}\left(w \widetilde{D}_{x}^{\frac{1}{2}} \nu\right)\right\|_{L_{x, T}^{2}}+\left\|P_{h i} \widetilde{D}_{x}^{s}\left(w \widetilde{D}_{x}^{\frac{1}{2}} \nu\right)\right\|_{L_{x}^{1} L_{T}^{2}} \\
\lesssim & T^{\frac{1}{2}}\left\|w \widetilde{D}_{x}^{\frac{1}{2}} \nu\right\|_{L_{x, T}^{2}}+\left\|D_{x}^{s} w\right\|_{L_{x}^{\frac{4}{3}} L_{T}^{4}}\left\|D_{x}^{\frac{1}{2}} v\right\|_{L_{x, T}^{4}}+\left\|w D_{x}^{s+\frac{1}{2}} \nu\right\|_{L_{x}^{1} L_{T}^{2}} \\
\lesssim & T\|v\|_{L_{T}^{\infty} H^{s}}^{3}+T^{\frac{1}{4}}\|v\|_{L_{x}^{2} L_{T}^{\infty}}\left\|D_{x}^{s} v\right\|_{L_{x, T}^{6}}^{\frac{3}{4}}\|v\|_{L_{T}^{\infty} H^{s}}^{\frac{1}{2}}\left\|D_{x}^{\frac{1}{2}} v\right\|_{L_{x, T}^{6}}^{\frac{3}{4}}+\|v\|_{L_{x}^{2} L_{T}^{\infty}}^{2}\left\|D_{x}^{s+\frac{1}{2}} v\right\|_{L_{x}^{\infty} L_{T}^{2}},
\end{aligned}
$$

where to get the second and third estimates, we have used Lemma 2.6(i),(iii), Bernstein, Hölder, and Sobolev's inequalities.

In order to deal with $F_{3,1}$, we rewrite it as

$$
F_{3,1}=P_{0}\left(v D_{x}\left(|v|^{2}\right)\right)=P_{0} D_{x}^{\frac{1}{2}}\left(v D_{x}^{\frac{1}{2}}\left(|v|^{2}\right)\right)-P_{0}\left(\left[D_{x}^{\frac{1}{2}}, v\right] D_{x}^{\frac{1}{2}}\left(|v|^{2}\right)\right) .
$$


Now from Bernstein, Hölder, and Sobolev's inequalities, we deduce

$$
\begin{aligned}
\left\|\int_{0}^{t} U(t-\tau) P_{0} D_{x}^{\frac{1}{2}}\left(v D_{x}^{\frac{1}{2}}\left(|v|^{2}\right)\right)(\tau) d \tau\right\|_{X_{T}^{s}} & \lesssim T\left\|v D_{x}^{\frac{1}{2}}\left(|v|^{2}\right)\right\|_{L_{T}^{\infty} L_{x}^{2}} \\
& \lesssim T\|v\|_{L_{T}^{\infty} H^{s}}\left\|D_{x}^{\frac{1}{2}}\left(|v|^{2}\right)\right\|_{L_{T}^{\infty} L_{x}^{2}} \\
& \lesssim T\|v\|_{L_{T}^{\infty} H^{s}}^{3} .
\end{aligned}
$$

Using similar arguments as above together with Lemma 2.6(v), we also obtain

$$
\begin{aligned}
\left\|\int_{0}^{t} U(t-\tau) P_{0}\left(\left[D_{x}^{\frac{1}{2}}, v\right] D_{x}^{\frac{1}{2}}\left(|v|^{2}\right)\right)(\tau) d \tau\right\|_{X_{T}^{s}} & \lesssim T^{\frac{1}{2}}\left\|\left[D_{x}^{\frac{1}{2}}, v\right] D_{x}^{\frac{1}{2}}\left(|v|^{2}\right)\right\|_{L_{x, T}^{2}} \\
& \lesssim T^{\frac{1}{2}}\left\|D_{x}^{\frac{1}{2}} v\right\|_{L_{x, T}^{6}}\left\|D_{x}^{\frac{1}{2}}\left(|v|^{2}\right)\right\|_{L_{x, T}^{3}} \\
& \lesssim T^{\frac{2}{3}}\left\|D_{x}^{\frac{1}{2}} v\right\|_{L_{x, T}^{6}}^{2}\|v\|_{L_{T}^{\infty} H^{s}}
\end{aligned}
$$

Gathering together (3.17) and (3.18), we get

$$
E_{3,1} \lesssim T\|v\|_{L_{T}^{\infty} H^{s}}^{3}+T^{\frac{2}{3}}\left\|D_{x}^{\frac{1}{2}} v\right\|_{L_{x, T}^{6}}^{2}\|v\|_{L_{T}^{\infty} H^{s}} .
$$

An application of Lemmas 2.4, 2.5 and 2.6(iv),(i) yields

$$
\begin{aligned}
& \left\|\int_{0}^{t} U(t-\tau) P_{ \pm h i}\left(v P_{\mp} \partial_{x}\left(|v|^{2}\right)\right)(\tau) d \tau\right\|_{X_{T}^{s}} \lesssim T^{\frac{1}{10}}\left\|D_{x}^{s-\frac{1}{10}} P_{ \pm}\left(v P_{\mp} \partial_{x}\left(|v|^{2}\right)\right)\right\|_{L_{x}^{\frac{5}{4}} L_{T}^{\frac{10}{7}}} \\
& \lesssim T^{\frac{1}{10}}\left\|D_{x}^{\frac{9}{10}} v\right\|_{L_{x}^{20} L_{T}^{20 / 9}}\left\|D_{x}^{S}\left(|v|^{2}\right)\right\|_{L_{x}^{\frac{4}{3}} L_{T}^{4}} \\
& \lesssim T^{\frac{1}{10}}\left\|D_{x}^{\frac{9}{10}} v\right\|_{L_{x}^{20} L_{T}^{20 / 9}}\|v\|_{L_{x}^{2} L_{T}^{\infty}}\left\|D_{x}^{s} v\right\|_{L_{x, T}^{4}} \\
& \lesssim T^{\frac{9}{40}}\left\|D_{x}^{\frac{9}{10}} v\right\|_{L_{x}^{20} L_{T}^{20 / 9}}\|v\|_{L_{x}^{2} L_{T}^{\infty}}\left\|D_{x}^{s} v\right\|_{L_{x, T}^{6}}^{\frac{3}{4}}\|v\|_{L_{T}^{\infty} H^{s}}^{\frac{1}{4}} .
\end{aligned}
$$

Thus,

$$
E_{3,2}+E_{3,3} \lesssim T^{\frac{9}{40}}\left\|D_{x}^{\frac{9}{10}} v\right\|_{L_{x}^{20} L_{T}^{20 / 9}}\|v\|_{L_{x}^{2} L_{T}^{\infty}}\left\|D_{x}^{s} v\right\|_{L_{x, T}^{6}}^{\frac{3}{4}}\|v\|_{L_{T}^{\infty} H^{s}}^{\frac{1}{4}} .
$$

In order to estimate $E_{4}$, we apply Bernstein and Sobolev's inequalities, and Lemmas 2.5 and 2.6 to arrive at

$$
\begin{aligned}
E_{4,1} \lesssim & T^{\frac{1}{2}}\left\|P_{0}\left(v \mathcal{H} P_{0}\left(\left[D_{x}^{\frac{1}{2}}, v\right] \widetilde{D}_{x}^{\frac{1}{2}} \bar{v}\right)\right)\right\|_{L_{x, T}^{2}}+T^{\frac{1}{2}}\left\|D_{x}^{s}\left(v \mathcal{H} P_{0}\left(\left[D_{x}^{\frac{1}{2}}, v\right] \widetilde{D}_{x}^{\frac{1}{2}} \bar{v}\right)\right)\right\|_{L_{x, T}^{2}} \\
\lesssim & T^{\frac{1}{2}}\left\{\left\|D_{x}^{s} v\right\|_{L_{x, T}^{6}}\left\|\left[D_{x}^{\frac{1}{2}}, v\right] \widetilde{D}_{x}^{\frac{1}{2}} \bar{v}\right\|_{L_{x, T}^{3}}+\|v\|_{L_{x, T}^{6}}\left\|\left[D_{x}^{\frac{1}{2}}, v\right] \widetilde{D}_{x}^{\frac{1}{2}} \bar{v}\right\|_{L_{x, T}^{3}}\right\} \\
\lesssim & T^{\frac{1}{2}}\left\|D_{x}^{s} v\right\|_{L_{x, T}^{6}}\left\|D_{x}^{\frac{1}{2}} v\right\|_{L_{x, T}^{6}}^{2}+T^{\frac{2}{3}}\|v\|_{L_{T}^{\infty} H^{s}}\left\|D_{x}^{\frac{1}{2}} v\right\|_{L_{x, T}^{6}}^{2}, \\
E_{4,2} \lesssim & \lesssim T^{\frac{1}{2}}\left\{\left\|P_{0}\left\{v P_{0} \widetilde{D}_{x}^{\frac{1}{2}}\left(v \widetilde{D}_{x}^{\frac{1}{2}} \bar{v}\right)\right\}\right\|_{L_{x, T}^{2}}+\left\|D_{x}^{s} P_{h i}\left\{v P_{0} \widetilde{D}_{x}^{\frac{1}{2}}\left(v \widetilde{D}_{x}^{\frac{1}{2}} \bar{v}\right)\right\}\right\|_{L_{x, T}^{2}}\right. \\
\lesssim & T^{\frac{1}{2}}\left\{\left\|D_{x}^{s} v\right\|_{L_{x, T}^{6}}\left\|P_{0} \widetilde{D}_{x}^{\frac{1}{2}}\left(v \widetilde{D}_{x}^{\frac{1}{2}} \bar{v}\right)\right\|_{L_{x, T}^{3}}+\|v\|_{L_{x, T}^{6}}\left(\left\|P_{0} D_{x}^{\frac{1}{2}}\left(v \widetilde{D}_{x}^{\frac{1}{2}} \bar{v}\right)\right\|_{L_{x, T}^{3}}\right.\right. \\
& \left.\left.+\left\|P_{0} D_{x}^{s+\frac{1}{2}}\left(v \widetilde{D}_{x}^{\frac{1}{2}} \bar{v}\right)\right\|_{L_{x, T}^{3}}\right)\right\} \\
& \lesssim T^{\frac{1}{2}}\left\|D_{x}^{s} v\right\|_{L_{x, T}^{6}}\left\|v \widetilde{D}_{x}^{\frac{1}{2}} \bar{v}\right\|_{L_{x, T}^{3}}+T^{\frac{2}{3}}\|v\|_{L_{T}^{\infty} H^{s}}\left\|v \widetilde{D}_{x}^{\frac{1}{2}} \bar{v}\right\|_{L_{x, T}^{3}} \\
\lesssim & T^{\frac{2}{3}}\|v\|_{L_{T}^{\infty} H^{s}}\left\|D_{x}^{s} v\right\|_{L_{x, T}^{6}}\left\|D_{x}^{\frac{1}{2}} v\right\|_{L_{x, T}^{6}}+T^{\frac{5}{6}}\|v\|_{L_{T}^{\infty} H^{s}}^{2}\left\|D_{x}^{\frac{1}{2}} v\right\|_{L_{x, T}^{6}}
\end{aligned}
$$


Therefore, from (3.21) and (3.22), we see that

$$
\begin{aligned}
E_{4} \lesssim & T^{\frac{1}{2}}\left\|D_{x}^{s} v\right\|_{L_{x, T}^{6}}\left\|D_{x}^{\frac{1}{2}} v\right\|_{L_{x, T}^{6}}^{2}+T^{\frac{2}{3}}\|v\|_{L_{T}^{\infty} H^{s}}\left\|D_{x}^{\frac{1}{2}} v\right\|_{L_{x, T}^{6}}\left\|D_{x}^{s} v\right\|_{L_{x, T}^{6}} \\
& +T^{\frac{2}{3}}\|v\|_{L_{T}^{\infty} H^{s}}\left\|D_{x}^{\frac{1}{2}} v\right\|_{L_{x, T}^{6}}^{2}+T^{\frac{5}{6}}\|v\|_{L_{T}^{\infty} H^{s}}^{2}\left\|D_{x}^{\frac{1}{2}} v\right\|_{L_{x, T}^{6}} .
\end{aligned}
$$

Next, to estimate the nonlinear terms without derivatives, it is enough to apply Lemmas 2.5, 2.3, and Hölder and Bernstein's inequalities to immediately obtain

$$
\sum_{j=6}^{11} E_{j}=\sum_{j=6}^{11}\left\|\int_{0}^{t} U(t-\tau) F_{j}(\tau) d \tau\right\|_{X_{T}^{s}} \lesssim T\|v\|_{L_{T}^{\infty} H^{s}}^{5} .
$$

Finally, we consider $E_{5}$. In view of Minkowski, Hölder, Bernstein, and Sobolev's inequalities, we deduce

$$
\begin{aligned}
& E_{5} \lesssim \int_{0}^{t}\left\|v \mathcal{H} P_{0}\left(\int_{-\infty}^{x} \mathcal{H} \partial_{y}\left(|v|^{2}\right)|v|^{2} d y\right)\right\|_{H^{s}}(\tau) d \tau \\
& \lesssim \int_{0}^{t}\|v\|_{H^{s}}\left\|\mathcal{H} P_{0}\left(\int_{-\infty}^{x} \mathcal{H} \partial_{y}\left(|v|^{2}\right)|v|^{2} d y\right)\right\|_{H^{s}}(\tau) d \tau \\
& \lesssim \int_{0}^{t}\|v\|_{H^{s}}\left\|P_{0}\left(\int_{-\infty}^{x} D_{y}^{\frac{1}{2}}\left(|v|^{2} D_{y}^{\frac{1}{2}}\left(|v|^{2}\right)\right) d y\right)\right\|_{H^{s}} \\
& +\int_{0}^{t}\|v\|_{H^{s}}\left\|P_{0}\left(\int_{-\infty}^{x}\left[D_{y}^{\frac{1}{2}},|v|^{2}\right] D_{y}^{\frac{1}{2}}\left(|v|^{2}\right) d y\right)\right\|_{H^{s}} \\
& \lesssim \int_{0}^{t}\|v\|_{H^{s}}\left\{\left\|P_{0} D_{x}^{\frac{1}{2}}\left(|v|^{2} D_{x}^{\frac{1}{2}}\left(|v|^{2}\right)\right)\right\|_{L_{x}^{2}}+\left\|P_{0}\left(\int_{-\infty}^{x}\left[D_{y}^{\frac{1}{2}},|v|^{2}\right] D_{y}^{\frac{1}{2}}\left(|v|^{2}\right) d y\right)\right\|_{H^{s}}\right\} \\
& \lesssim T\|v\|_{L_{T}^{\infty} H^{s}}\left\||v|^{2} D_{x}^{\frac{1}{2}}\left(|v|^{2}\right)\right\|_{L_{T}^{\infty} L_{x}^{2}}+\int_{0}^{t}\|v\|_{H^{s}}\left\|\left[D_{x}^{\frac{1}{2}},|v|^{2}\right] D_{x}^{\frac{1}{2}}\left(|v|^{2}\right)\right\|_{L_{x}^{2}} \\
& \lesssim T\|v\|_{L_{T}^{\infty} H^{s}}^{5}+T^{\frac{1}{2}}\|v\|_{L_{T}^{\infty} H^{s}}\left\|\left[D_{x}^{\frac{1}{2}},|v|^{2}\right] D_{x}^{\frac{1}{2}}\left(|v|^{2}\right)\right\|_{L_{x, T}^{2}} .
\end{aligned}
$$

Now, applying Lemma 2.6 (ii),(i) and Holder's inequality to the last term in (3.25), we obtain

$$
\begin{aligned}
\left\|\left[D_{x}^{\frac{1}{2}},|v|^{2}\right] D_{x}^{\frac{1}{2}}\left(|v|^{2}\right)\right\|_{L_{x, T}^{2}} & \lesssim\left\|D_{x}^{\frac{1}{2}}\left(|v|^{2}\right)\right\|_{L_{x, T}^{4}}^{2} \lesssim\|v\|_{L_{x, T}^{12}}^{2}\left\|D_{x}^{\frac{1}{2}} v\right\|_{L_{x, T}^{6}}^{2} \\
& \lesssim T^{\frac{1}{6}}\|v\|_{L_{T}^{\infty} H^{s}}^{2}\left\|D_{x}^{\frac{1}{2}} v\right\|_{L_{x, T}^{6}}^{2} .
\end{aligned}
$$

Therefore, (3.25) and (3.26) lead to

$$
E_{5} \lesssim T\|v\|_{L_{T}^{\infty} H^{s}}^{5}+T^{\frac{2}{3}}\|v\|_{L_{T}^{\infty} H^{s}}^{3}\left\|D_{x}^{\frac{1}{2}} v\right\|_{L_{x, T}^{6}}^{2} .
$$

Thus, from (3.11), (3.14), (3.15), (3.19), (3.20), (3.23), (3.24) and (3.27), we see that

$$
\begin{gathered}
\|\Phi(v)\|_{X_{T}^{s}} \lesssim\|\psi\|_{H^{s}}+\left(1+T^{\frac{3}{20}}+T^{\frac{9}{40}}+T^{\frac{1}{4}}+T^{\frac{1}{2}}+T^{\frac{2}{3}}+T^{\frac{5}{6}}+T\right)\|v\|_{X_{T}^{s}}^{3} \\
+\left(T^{\frac{2}{3}}+T\right)\|v\|_{X_{T}^{s}}^{5} \\
\leq c\|\psi\|_{H^{s}}+c\left(1+T^{\frac{3}{20}}\right)\|v\|_{X_{T}^{s}}^{3}+c T^{\frac{2}{3}}\|v\|_{X_{T}^{s}}^{5} .
\end{gathered}
$$


Fix $\delta=1 /(8 c \sqrt{c})$ and take $a=a\left(\|\psi\|_{H^{s}}\right)>0$ such that $a \in\left(2 c\|\psi\|_{H^{s}}, 4 c\|\psi\|_{H^{s}}\right)$. It follows that, if we choose $T>0$ such that $c T^{\frac{3}{20}}\left(4 c\|\psi\|_{H^{s}}\right)^{2}+c T^{\frac{2}{3}}\left(4 c\|\psi\|_{H^{s}}\right)^{4} \leq \frac{1}{4}$, then we get $\Phi(v) \in X_{T}^{s}(a)$. Similar arguments show that $\Phi$ is a contraction. Since the remainder of the proof follows standard arguments, we ommit the details (see, for example, [16, Theorem 4.1] or [7, Theorem 2.1]).

3.3. Proof of Theorem 1.1. With Proposition 3.1 in hand, we are in a position to prove Theorem 1.1. Let $1 / 2<s<9 / 10$ and fix $\phi \in H^{s}$ with $\|\phi\|_{H^{s}} \ll 1$. Define $\psi$ as the following:

$$
\psi(x)=e^{i \rho(\phi)} \phi(x), \quad \text { where } \quad \rho(\phi)=-\frac{1}{2} \lambda \mathcal{H} P_{0}\left(\int_{-\infty}^{x}|\phi(y)|^{2} d y\right) .
$$

By using Lemma 2.6(v), Bernstein's inequality, and Sobolev's embedding, we see that

$$
\begin{aligned}
\left\|D_{x}^{s} \psi\right\|_{L^{2}} & =\left\|D_{x}^{s}\left(e^{i \rho(\phi)} \phi\right)\right\|_{L^{2}} \\
& \lesssim\left\|e^{i \rho(\phi)}\right\|_{L^{\infty}}\left\|D_{x}^{s} \phi\right\|_{L^{2}}+\|\phi\|_{L^{\infty}}\left\|D_{x}^{s}\left(e^{i \rho(\phi)}\right)\right\|_{L^{2}} \\
& \lesssim\left\|D_{x}^{s} \phi\right\|_{L^{2}}+\|\phi\|_{H^{s}}+\|\phi\|_{H^{s}}\left\|P_{h i} \partial_{x}\left(e^{i \rho(\phi)}\right)\right\|_{L^{2}} \\
& \lesssim\left(1+\|\phi\|_{H^{s}}^{2}\right)\|\phi\|_{H^{s}} .
\end{aligned}
$$

Moreover, since $\|\phi\|_{L^{2}}=\|\psi\|_{L^{2}}$, we obtain that $\psi \in H^{s}$ and $\|\psi\|_{H^{s}} \ll 1$. According to Proposition 3.1, there exists a unique solution $\left.v \in C([-T, T]) ; H^{s}(\mathbb{R})\right)$ for the IVP (3.8). Let $\phi^{(n)}$ be a sequence in $H^{\infty}$ such that $\phi^{(n)} \rightarrow \phi$ in $H^{s}$, and let $v^{(n)}$ be the solution of (3.8) with initial data

$$
\psi^{(n)}(x)=e^{i \rho\left(\phi^{(n)}\right)} \phi^{(n)}(x) .
$$

Similarly to (3.29), we see that

$$
\left\|\psi^{(n)}-\psi\right\|_{H^{s}} \lesssim\left(1+\|\phi\|_{H^{s}}^{2}+\left\|\phi^{(n)}\right\|_{H^{s}}^{2}\right)\left\|\phi^{(n)}-\phi\right\|_{H^{s}} .
$$

Hence $\psi^{(n)} \rightarrow \psi$ in $H^{s}$. From the continuity of the flow map given in Proposition 3.1, we obtain that $v^{(n)} \rightarrow v$ in $\left.C([-T, T]) ; H^{s}(\mathbb{R})\right)$.

Next, define

$$
\begin{aligned}
& u^{(n)}(x, t)=e^{-i \rho\left(v^{(n)}\right)} v^{(n)}(x, t), \\
& u(x, t)=e^{-i \rho(v)} v(x, t) .
\end{aligned}
$$

As in (3.30), we have

$$
\left\|u^{(n)}-u\right\|_{L_{T}^{\infty} H^{s}} \lesssim\left(1+\|v\|_{L_{T}^{\infty} H^{s}}^{2}+\left\|v^{(n)}\right\|_{L_{T}^{\infty} H^{s}}^{2}\right)\left\|v^{(n)}-v\right\|_{L_{T}^{\infty} H^{s}}
$$

which implies that $u^{(n)} \rightarrow u$ in $C\left([-T, T] ; H^{s}(\mathbb{R})\right)$. We claim that $u$ solves the IVP associated with (1.2) with initial data $\phi$. Indeed, obviously for $t \in[-T, T], u^{(n)}(t)$ solves the integral equation

$$
u^{(n)}(t)=U(t) \phi^{(n)}+\int_{0}^{t} U(t-\tau)\left(\partial_{x}\left(\left|u^{(n)}\right|^{2} u^{(n)}\right)+\lambda \partial_{x}\left(\mathcal{H}\left(\left|u^{(n)}\right|^{2}\right) u^{(n)}\right)\right)(\tau) d \tau .
$$


But, from the Sobolev embedding, we deduce

$$
\begin{aligned}
&\left\|\int_{0}^{t} U(t-\tau) \partial_{x}\left(\left|u^{(n)}\right|^{2} u^{(n)}-|u|^{2} u\right)(\tau) d \tau\right\|_{H^{-1}} \\
& \lesssim\left(\|u\|_{L_{T}^{\infty} H^{s}}^{2}+\left\|u^{(n)}\right\|_{L_{T}^{\infty} H^{s}}^{2}\right)\left\|u^{(n)}-u\right\|_{L_{T}^{1} L^{2} .}
\end{aligned}
$$

Furthermore, using that $i \mathcal{H}=P_{+}-P_{-}, 1=P_{+}+P_{-}$, and the fact that $P_{+}$is bounded on $L^{2}$, we have

$$
\begin{aligned}
&\left\|\int_{0}^{t} U(t-\tau) \partial_{x}\left(\mathcal{H}\left(\left|u^{(n)}\right|^{2}\right) u^{(n)}-\mathcal{H}\left(|u|^{2}\right) u\right)(\tau) d \tau\right\|_{H^{-1}} \\
& \lesssim\left(\|u\|_{L_{T}^{\infty} H^{s}}^{2}+\left\|u^{(n)}\right\|_{L_{T}^{\infty} H^{s}}^{2}\right)\left\|u^{(n)}-u\right\|_{L_{T}^{1} H^{s}} .
\end{aligned}
$$

Therefore, by letting $n \rightarrow \infty$ in (3.32), we deduce that (3.32) holds with $u^{(n)}$ and $\phi^{(n)}$ replaced by $u$ and $\phi$, respectively.

The uniqueness of $u$ follows from that for $v$. In addition, the continuous dependence follows from similar arguments as above.

Finally, in order to show that it is enough to take $\|\phi\|_{L^{2}} \ll 1$ instead of $\|\phi\|_{H^{s}} \ll 1$, we use the scaling in (1.5). Indeed, $u(x, t)$ is the solution of the IVP for $(1.2)$ on $[0, T]$ with initial data $\phi \in H^{s}(\mathbb{R})$ if and only if $u_{\sigma}(x, t)=\sigma^{\frac{1}{2}} u\left(\sigma x, \sigma^{2} t\right)$ is the solution on $\left[0, \sigma^{-2} T\right]$ with data $\phi_{\sigma}=\sigma^{\frac{1}{2}} \phi(\sigma x)$ in $H^{s}(\mathbb{R})$. From (1.6), we have that

$$
\left\|\phi_{\sigma}\right\|_{H^{s}} \leq\|\phi\|_{L^{2}}+\sigma^{s}\left\|D_{x}^{s} \phi\right\|_{L^{2}}
$$

and so, taking $\|\phi\|_{L^{2}} \ll 1$ and $\sigma=o\left(\|\phi\|_{H^{s}}^{-2}\right)$ we obtain $\left\|\phi_{\sigma}\right\|_{H^{s}} \ll 1$. This completes the proof of Theorem 1.1.

\section{Proof of Theorem 1.4}

The aim of this section is to prove Theorem 1.4. The proof is close to that of Theorem 7.1 in [28]. We begin with the following proposition.

Proposition 4.1. Let $s<1 / 2$ and $T>0$. Then there does not exist any space $X_{T}^{s}$ such that $X_{T}^{s}$ is continuously embedded in $C\left([-T, T] ; H^{s}(\mathbb{R})\right)$, i.e.

$$
\|u\|_{C\left([-T, T] ; H^{s}\right)} \lesssim\|u\|_{X_{T}^{s}}, \quad \forall u \in X_{T}^{s},
$$

and such that

$$
\|U(t) \phi\|_{X_{T}^{s}} \lesssim\|\phi\|_{H^{s}}, \quad \forall \phi \in H^{s}(\mathbb{R})
$$

and

$$
\begin{aligned}
& \quad\left\|\int_{0}^{t} U(t-\tau)\left[\partial_{x}(u(\tau) v(\tau) \overline{w(\tau)})+\lambda \partial_{x}(u(\tau) \mathcal{H}(v(\tau) \overline{w(\tau)}))\right] d \tau\right\|_{X_{T}^{s}} \\
& \lesssim\|u\|_{X_{T}^{s}}\|v\|_{X_{T}^{s}}\|w\|_{X_{T}^{s}},
\end{aligned}
$$

for all $u, v, w \in X_{T}^{s}$.

Proof. Let $s<1 / 2$. From now on, we shall use the notation

$$
\partial_{x}\left(|U(\tau) \phi|^{2} U(\tau) \phi\right)+\partial_{x}\left(\mathcal{H}\left(|U(\tau) \phi|^{2}\right) U(\tau) \phi \equiv F(U(t) \phi) .\right.
$$


Suppose that there exists a space $X_{T}^{s}$ satisfying (4.1)-(4.3) for all $u, v, w \in X_{T}^{s}$, and fix $0<t<T$. Taking $\phi \in H^{s}(\mathbb{R})$ and using (4.1)-(4.3), with $u(t)=v(t)=w(t)=U(t) \phi$, we see that

$$
\left\|\int_{0}^{t} U(t-\tau) F(U(t) \phi) d \tau\right\|_{H^{s}} \lesssim\|\phi\|_{H^{s}}^{3} .
$$

Next, we show that (4.4) fails for an appropriate choice of $\phi$. This would lead us to a contradiction.

Choose the following $\phi$, defined by its Fourier transform,

$$
\widehat{\phi}(\xi)=N^{-s} \chi_{I}(\xi),
$$

where $\chi_{I}$ is the characteristic function of the interval $I=[N-\eta, N+\eta]$, with $N \gg 1$ and $0<\eta \ll 1$. Note that $\|\phi\|_{H^{s}} \sim 1$.

Now, by using the definitions of $U(t)$ and $\phi$, we can write

$$
\begin{aligned}
& \mathcal{F}\left\{\int_{0}^{t} U(t-\tau) F(U(t) \phi) d \tau\right\}(\xi) \\
= & \frac{\xi e^{-i t \xi^{2}}}{N^{3 s}} \int_{\Omega(\xi)} \frac{e^{2 i t\left(\xi-\xi_{1}\right)\left(\xi_{1}+\xi_{2}\right)}-1}{2 t\left(\xi-\xi_{1}\right)\left(\xi_{1}+\xi_{2}\right)}\left(1-i \operatorname{sgn}\left(\xi-\xi_{1}\right)\right) d \xi_{1} d \xi_{2},
\end{aligned}
$$

where

$$
\Omega(\xi)=\left\{\left(\xi_{1}, \xi_{2}\right) \in \mathbb{R}^{2} / \xi_{1},-\xi_{2}, \xi-\xi_{1}-\xi_{2} \in I\right\} .
$$

Observe that when $\left(\xi_{1}, \xi_{2}\right) \in \Omega(\xi)$ we have

$$
\xi \sim N \quad \text { and } \quad\left|\left(\xi-\xi_{1}\right)\left(\xi_{1}+\xi_{2}\right)\right| \lesssim \eta^{2} .
$$

For any $\xi \in(N-\eta, N+\eta)$ we have that $|\Omega(\xi)| \gtrsim \eta^{2}$. Moreover, a simple analysis shows that

$$
\operatorname{supp} \mathcal{F}\left\{\int_{0}^{t} U(t-\tau) F(U(t) \phi) d \tau\right\} \subseteq[N-3 \eta, N+3 \eta]
$$

Finally, choosing $\eta$ small enough, we deduce that

$$
\frac{e^{2 i t\left(\xi_{1}+\xi_{3}\right)\left(\xi_{2}+\xi_{3}\right)}-1}{\left(\xi_{1}+\xi_{3}\right)\left(\xi_{2}+\xi_{3}\right)}=2 i t+o(\eta)
$$

which in turns implies that

$$
\left\|\int_{0}^{t} U(t-\tau) F(U(t) \phi) d \tau\right\|_{H^{s}} \gtrsim N^{1-2 s} .
$$

Therefore, gathering together (4.4) and (4.8), we conclude that

$$
N^{1-2 s} \lesssim 1, \quad \forall N \gg 1,
$$

which is a contradiction since $s<1 / 2$.

Remark 4.2. As is well known, a consequence of Proposition 4.1 is that it is not possible to solve the IVP $(1.2)$ in $H^{s}(\mathbb{R})$ using a Fixed Point Theorem if $s<1 / 2$. 
Proof of Theorem 1.4.

Proof. Consider the Cauchy problem for (1.2) with initial data $u(x, 0)=\mu \phi(x)$. Suppose that $u=u(x, t ; \mu)$ is the local solution of that IVP and that its flow-map solution

$$
S: H^{s}(\mathbb{R}) \longrightarrow C\left([0, T] ; H^{s}(\mathbb{R})\right)
$$

is $C^{3}$-differentiable at the origin. The associated integral equation is

$$
u(t)=U(t) \mu \phi+\int_{0}^{t} U(t-\tau) F(U(\tau) \phi) d \tau .
$$

Differentiating (4.9) with respect to $\mu$ and evaluating at $\mu=0$, we have

$$
\left.\frac{\partial u}{\partial \mu}\right|_{\mu=0}=U(t) \phi,\left.\quad \frac{\partial^{2} u}{\partial \mu^{2}}\right|_{\mu=0}=0, \text { and }\left.\frac{\partial^{3} u}{\partial \mu^{3}}\right|_{\mu=0}=6 \int_{0}^{t} U(t-\tau) F(U(\tau) \phi) d \tau .
$$

By the hypothesis of $C^{3}$-differentiability, we then obtain

$$
\left\|\int_{0}^{t} U(t-\tau) F(U(\tau) \phi) d \tau\right\|_{H^{s}} \lesssim\|\phi\|_{H^{s}}^{3}
$$

which is estimate (4.4). But, from Proposition 4.1, it is false for $s<1 / 2$.

\section{Proof of Theorem 1.5}

In this section we prove Theorem 1.5. The proof follows from standard techniques because the nDNLS equation does not conserve energy.

We first observe that to formally obtain (1.4), we multiply (1.2) by $\bar{u}$ and integrate over $\mathbb{R}$. Taking the real part of the obtained equation and integrating by parts gives (1.4).

Assume next that (1.2) admits a traveling-wave solution of the form (1.10) satisfying the hypotheses of Theorem 1.5. Then

$$
E\left(u_{\omega, c}(t)\right)=\int_{-\infty}^{\infty}|\zeta(x)|^{2} d x
$$

Since the right-hand side of (5.1) does not depend on $t$, it follows that

$$
\frac{d}{d t} E\left(u_{\omega, c}(t)\right)=0
$$

This last equality contradicts (1.4) for any $\lambda \neq 0$. This completes the proof of the theorem.

Acknowledgement. R.P. de Moura was supported by $\mathrm{CNPq} / \mathrm{Brazil}$ grant 151652/2007-4 and A. Pastor was supported by CNPq/Brazil grant 152234/2007-1. This paper was written when both authors had post-doctorial positions at IMPABrazil. They gratefully acknowledge IMPA's hospitality. The authors also acknowledge the referees for helpful comments and suggestions, and Jaime Angulo for helpful discussions. 


\section{REFERENCES}

[1] H. Biagioni and F. Linares, Ill-posedness for the derivative Schrödinger and generalized Benjamin-Ono equations, Trans. Amer. Math. Soc., 353, 3649-3659, 2001.

[2] J. Bourgain, Periodic Korteweg-de Vries equation with measures as initial data, Selecta Math., 3, 115-159, 1997.

[3] M. Colin and M. Ohta, Stability of solitary waves for derivative nonlinear Schrödinger equation, Ann. Inst. H. Poincaré Anal. Non Linéaire, 23, 753-764, 2006.

[4] J. Colliander, M. Keel, G. Staffilani, H. Takaoka and T. Tao, Global well-posedness for Schrödinger with derivative, SIAM J. Math. Anal., 33, 649-669, 2001.

[5] J. Colliander, M. Keel, G. Staffilani, H. Takaoka and T. Tao, A refined global well-posedness result for Schrödinger with derivative, SIAM J. Math. Anal., 34, 64-86, 2002.

[6] M. Christ and A. Kiselev, Maximal functions associated to filtrations, J. Funct. Anal., 179, 409-425, 2001.

[7] R.P. de Moura, Well-posedness for the nonlocal nonlinear Schrödinger equation, J. Math. Anal. Appl., 326, 1254-1267, 2007.

[8] T. Flå, E. Mjølhus and J. Wyller, Nonlinear Landau damping of weakly dispersive circularly polarized MHD waves, Phys. Scr., 40, 219-226, 1989.

[9] A. Grünrock, Bi- and trilinear Schrödinger estimates in one space dimension with applications to cubic NLS and DNLS, Int. Math. Res. Not., 2005, 2525-2558, 2005.

[10] A. Grünrock and S. Herr, Low regularity well-posedness of the derivative nonlinear Schrödinger equation with periodic initial data, SIAM J. Math. Anal., 39, 1890-1920, 2008.

[11] B. Guo and Y. Wu, Orbital stability for the nonliear derivative Schrödinger equation, J. Diff. Eqs., 123, 55-35, 1995.

[12] N. Hayashi and T. Ozawa, On the derivative nonlinear Schrödinger equation, Physica D, 55, 14-36, 1992.

[13] N. Hayashi and T. Ozawa, Finite energy solutions of nonlinear Schrödinger equations of derivative type, SIAM J. Math. Anal., 25, 1488-1503, 1994.

[14] S. Herr, On the Cauchy problem for the derivative nonlinear Schrödinger equation with periodic boundary condition, Int. Math. Res. Not., 2006, 1-33, 2006.

[15] C.E. Kenig, G. Ponce and L. Vega, Oscillatory integrals and regularity of dispersive equations, Indiana Univ. Math. J., 40, 33-69, 1991.

[16] C.E. Kenig, G. Ponce and L. Vega, Small solutions to nonlinear Schrödinger equations, Ann. Inst. H. Poincaré Anal. Non Linéaire, 10, 255-288, 1993.

[17] C.E. Kenig, G. Ponce and L. Vega, Well-posedness of the initial value problem for the Kortewegde Vries equation, J. Amer. Math. Soc., 4, 323-347, 1991.

[18] C. E. Kenig, G. Ponce and L. Vega, Well-Posedness and scattering Results for the generalized Korteweg-de Vries equation via the contraction principle, Commun. Pure Appl. Math., 46, 527-620, 1993.

[19] E. Mjølhus, On the modulational instability of hydromagnetic waves parallel to the magnetic field, J. Plasma Phys., 16, 321-334, 1976.

[20] E. Mjølhus and J. Wyller, Nonlinear Alfvén waves in a finite-beta plasma, J. Plasma Phys., 40, 299-318, 1988.

[21] E. Mjølhus and J. Wyller, Alfvén solitions, Phys. Scr., 33, 442-451, 1986.

[22] L. Molinet and F. Ribaud, Well-posedness results for the generalized Benjamin-Ono equation with arbitrary initial data, Int. Math. Res. Not., 2004, 3757-3795, 2004.

[23] L. Molinet, J.-C. Saut, and N. Tzvetkov, Ill-posedness issues for the Benjamin-Ono and related equations, SIAM J. Math. Anal., 33, 982-988, 2001.

[24] T. Ozawa, On the nonlinear Schrödinger equations of derivative type, Indiana Univ. Math. J., 45, 137-163, 1996.

[25] T. Ozawa and Y. Tsutsumi, Space-time estimates for null gauge forms and nonlinear Schrödinger equations, Differential Integral Equations, 11, 201-222, 1998.

[26] D. Rial, O Problema de Cauchy Para Equações de Schrödinger Quase-lineares, Ph.D. Thesis, IMPA, 1992.

[27] D. Rial, Weak solutions for the derivative nonlinear Schrödinger equation, Nonlinear Anal., 49, 149-158, 2002.

[28] H. Takaoka, Global well-posedness for Schrödinger equations with derivative in a nonlinear term and data in low-order Sobolev spaces, Electron. J. Diff. Eqs., 2001, 1-23, 2001.

[29] H. Takaoka, Well-posedness for the one-dimensional nonlinear Schrödinger equation with the derivative nonlinearity, Adv. Diff. Eqs., 4, 561-580, 1999.

[30] N. Tzvetkov, Remarks on local ill-posedness for KdV equation, C. R. Acad. Sci. Paris Sér. I Math., 329, 1043-1047, 1999. 
[31] W. van Saarloos and P.C. Hohenberg, Fronts, pulses, sources and sinks in generalized complex Ginzburg-Landau equation, Physica D, 56, 303-367, 1992.

[32] J. Wyller, T. Flå and E. Mjølhus, The effect of resonant particles on Alfvén solitons, Physica D, 39, 405-422, 1989. 